(1)

CrossMark

\title{
Asthma in reproductive-aged women with polycystic ovary syndrome and association with obesity
}

\author{
Thaw D. Htet ${ }^{1}$, Helena J. Teede ${ }^{1,2,3,4}$, Barbora de Courten ${ }^{1,2,3}$, Deborah Loxton ${ }^{5}$, \\ Francisco G. Real ${ }^{6,7}$, Lisa J. Moran ${ }^{1,3,4}$ and Anju E. Joham ${ }^{1,2,3}$
}

Affiliations: ${ }^{1}$ Monash Centre for Health Research and Implementation, School of Public Health and Preventive Medicine, Monash University, Clayton, Australia. ${ }^{2}$ Diabetes and Vascular Medicine Unit, Monash Health, Clayton, Australia. ${ }^{3}$ Monash Partners Academic Health Sciences Centre, Clayton, Australia. ${ }^{4}$ The Robinson Research Institute, University of Adelaide, North Adelaide, Australia. ${ }^{5}$ Research Centre for Gender, Health and Ageing, University of Newcastle, Callaghan, Australia. ${ }^{6}$ Dept of Clinical Science, University of Bergen, Bergen, Norway. ${ }^{7}$ Dept of Gynecology and Obstetrics, Haukeland University Hospital, Bergen, Norway.

Correspondence: Anju E. Joham, Monash Centre for Health Research and Implementation, School of Public Health and Preventive Medicine, Monash University, Locked Bag 29, Monash Medical Centre, Clayton, Victoria 3168, Australia. E-mail: anju.johamamonash.edu

@ERSpublications

In reproductive-aged women, polycystic ovary syndrome is associated with asthma independent of BMI http://ow.ly/YSa330a0vMm

Cite this article as: Htet TD, Teede HJ, de Courten B, et al. Asthma in reproductive-aged women with polycystic ovary syndrome and association with obesity. Eur Respir J 2017; 49: 1601334 [https://doi.org/ 10.1183/13993003.01334-2016].

ABSTRACT Recent research suggests that women with polycystic ovary syndrome (PCOS) may have a higher prevalence of asthma. However, there are no epidemiological studies aimed primarily at exploring the relationship between PCOS and asthma, and the effect of body mass index (BMI) on this association.

This study is a cross-sectional analyses of data from the Australian Longitudinal Study on Women's Health, a large, community-based, prospective study to examine the association between PCOS and asthma in women aged $28-33$ years ( $n=478$ PCOS and $n=8134$ controls).

The prevalence of asthma was $15.2 \%$ in women with PCOS and $10.6 \%$ in women without PCOS $(p=0.004)$. Women with PCOS who had asthma had a trend for a higher BMI compared with women without asthma $\left(29.9 \pm 0.9\right.$ versus $\left.27.7 \pm 0.4 \mathrm{~kg} \cdot \mathrm{m}^{-2} ; \mathrm{p}=0.054\right)$. Women without PCOS who had asthma had a higher BMI compared with women without asthma $\left(26.4 \pm 0.2\right.$ versus $24.9 \pm 0.1 \mathrm{~kg} \cdot \mathrm{m}^{-2}$; $\left.\mathrm{p}<0.001\right)$. After adjusting for age, BMI and smoking status, PCOS was associated with increased odds of asthma (odds ratio $1.34,95 \%$ CI 1.004-1.79; $\mathrm{p}=0.047$ ).

This study showed both PCOS status and overweight/obese status were independently associated with asthma. Further prospective studies are required to explore the possible mechanisms underpinning the association between asthma and PCOS.

Received: July 052016 | Accepted after revision: March 092017

Support statement: This research received no specific grant from any funding agency in the public, commercial or not-for-profit sectors. H.J. Teede is a National Health and Medical Research Council of Australia (NHMRC) Practitioner Fellow. The Australian Longitudinal Study on Women's Health is funded by the Australian Government Dept of Health. B. de Courten is supported by National Heart Foundation (NHF) Future Leader Fellowship 1000864 from the NHF. F.G. Real is supported by a post-doctoral fellowship grant from the University of Bergen, Norway. L.J. Moran is supported by a South Australian Cardiovascular Research Development Program Fellowship; a programme collaboratively funded by the NHF, the South Australian Dept of Health, and the South Australian Health and Medical Research Institute. A.E. Joham is a NHMRC Early Career Fellowship holder.

Conflict of interest: None declared.

Copyright CERS 2017 


\section{Introduction}

Polycystic ovary syndrome (PCOS) is an endocrine disorder which is becoming increasingly more common in women of reproductive age, with a prevalence varying from $9 \%$ to $18 \%$ [1]. The exact prevalence is difficult to establish due to the use of different diagnostic criteria and variations in study populations [1-4]. Currently, PCOS is defined by the 2003 Rotterdam criteria, which requires two out of three criteria: oligo/anovulation, clinical and/or biochemical hyperandrogenism and ultrasound evidence of polycystic ovaries, and exclusion of other causes of androgen excess or ovulation disorders [3, 4]. Women with PCOS have reproductive health issues, e.g. irregular periods and infertility, as well as metabolic complications, e.g. obesity, dyslipidaemia, hypertension, insulin resistance and type 2 diabetes mellitus [5-9]. Women can present with varying degrees of clinical symptoms and biochemical heterogeneity, leading to a wide range of phenotypes with significant negative impact on physical and psychological quality of life [5-9].

Asthma is a recurrent, reversible bronchial obstruction, and is associated with bronchial hyperresponsiveness and evidence of chronic airway inflammation [10]. The disease can start at any age and it may be either atopic or nonatopic [10]. It is one of the most common medical conditions, affecting $8-10 \%$ of individuals in the community, is chronic and debilitating, and is a significant burden on health-related expenditure [10-12]. The pathogenesis of asthma is complex and multifactorial, including genetic, immunological and inflammatory mechanisms, and several factors such as smoking, inhalant allergens, exercise and urbanisation have been identified as contributing to asthma pathogenesis [10-12]. Current treatment strategies are through environmental control and noncurative pharmacological therapies, and management can be challenging due to medication adherence [10]. Literature on preventive strategies for asthma is limited and due to the temporal trend in asthma prevalence, asthma remains a significant burden on healthcare systems worldwide $[11,12]$.

A recent Danish register-based study showed increased asthma prevalence in women with PCOS compared with controls ( $3 \%$ versus $2.2 \%$; $\mathrm{p}<0.001$ ) [13]. Women with PCOS are recognised as having a higher risk of increased obesity and central obesity with a pooled estimated prevalence of $61 \%$ [14]. Epidemiological studies have previously described a link between obesity and asthma, but it is uncertain whether the increased prevalence of asthma in PCOS is primarily related to obesity [10, 15, 16]. Nonobese women with PCOS have metabolic risk factors similar to obese women with PCOS, suggesting that increased body mass index (BMI) is unlikely to be the sole causative factor for PCOS-related metabolic complications $[9,17]$. Prior research has also shown that menstrual irregularities are associated with reduced lung function and increased severity of asthma, suggesting a possible hormonal component to the pathogenesis of asthma [18]. There has also been conflicting evidence of a possible relationship between the oral contraceptive pill (OCP) use and asthma, and a possible relationship between exogenous sex hormones use and asthma and asthmatic symptoms [18-20]. Therefore, it is important to ascertain whether PCOS, with its complex underlying mechanisms, is an independent risk factor for asthma. However, there are no previous community-based studies with the primary aim of exploring the associations between asthma and PCOS, and the effect of BMI on this relationship.

We hypothesise that women with PCOS have increased incidence of asthma which may or may not be related to obesity. Therefore, the aim of this study was to examine the self-reported prevalence of asthma in women with and without PCOS in the general population, and to further determine the effect of BMI on this relationship.

\section{Methods}

The Australian Longitudinal Study on Women's Health (ALSWH) is a longitudinal, prospective study of women based in the community who were randomly selected from the National Health Insurance Scheme, the Medicare database which consists of all Australian citizens and permanent residents. The first survey was mailed in 1996 to women of three different age groups: aged 18-23, 45-50 and 70-75 years at that time. There was intentional oversampling from rural and remote populations to ensure that these areas were adequately represented [21]. The study's initial aims were to investigate the demographics, social, psychological and physical behaviour of Australian women and their impact on self-reported health issues with a follow-up survey being sent every 3 years.

Using the cohort of women born from 1973 to 1978, this study analysed data collected at Survey 4 which was conducted in 2006. In 1996, this cohort originally included 14247 women, of whom 9145 (62\%) were still participating at Survey 4. Data was analysed from 8612 respondents who responded to the question on PCOS (figure 1). The women were aged between 28 and 33 years at Survey 4.

All study methods were approved by the Human Research Ethics Committees of the University of Newcastle (Callaghan, Australia) and University of Queensland (St Lucia, Australia). 
FIGURE 1 Consort diagram of the cohort studied. ALSWH: Australian Longitudinal Study on Women's Health; PCOS: polycystic ovary syndrome; BMI: body mass index.

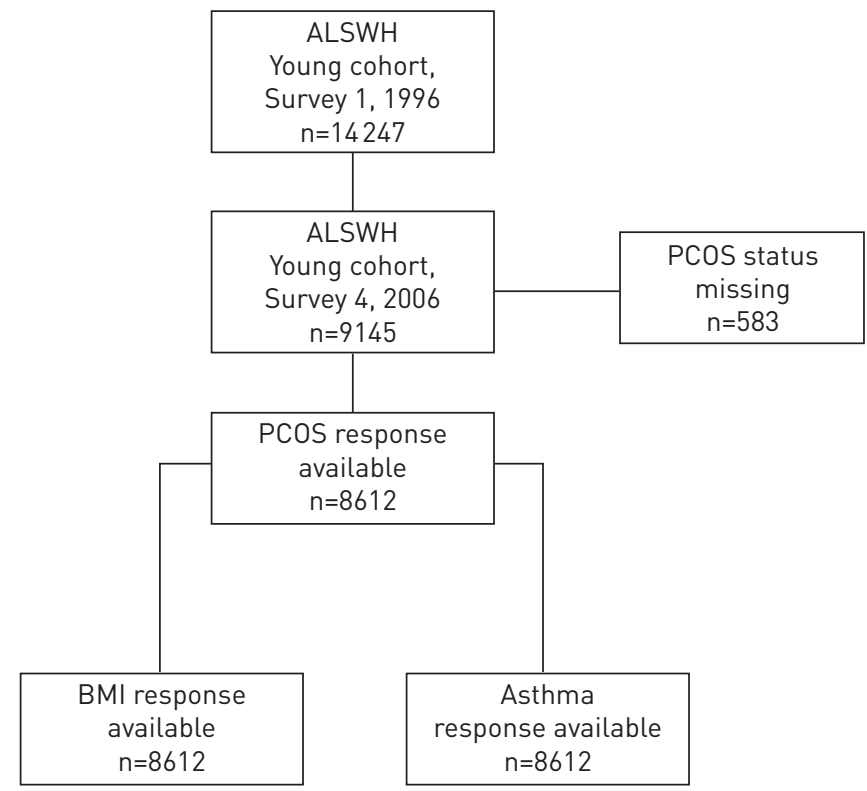

\section{Measures}

Outcome variable

The outcome variable was asthma. Women were asked "In the last 3 years, have you been diagnosed or treated for asthma?". Women who stated "Yes" in Survey 4 were considered to have asthma.

\section{Explanatory variables}

The explanatory variables were PCOS, BMI, OCP use and smoking.

Women were asked "In the last 3 years, have you been diagnosed or treated for Polycystic Ovary Syndrome?". Women who answered "Yes" were regarded as having PCOS.

BMI was calculated from self-reported height and weight, and classified according to World Health Organization criteria as normal weight (BMI 18.5-24.9 $\mathrm{kg} \cdot \mathrm{m}^{-2}$ ), overweight (BMI 25.0-29.9 $\mathrm{kg} \cdot \mathrm{m}^{-2}$ ) and obese (BMI $\left.>30.0 \mathrm{~kg} \cdot \mathrm{m}^{-2}\right)$.

Women who answered "Yes" to OCP use of $\geqslant 2$ years were defined as having used the OCP.

Women were asked "How often do you currently smoke cigarettes or any tobacco products?". Women who answered "Yes" to "Daily", "At least weekly (but not daily)" and "Less often than weekly" were considered to be smokers.

\section{Statistical analyses}

Data are presented as mean \pm SE (continuous explanatory variables) or percentages (categorical explanatory variables). Differences in variables at baseline between subgroups of the study population were assessed using univariable regression or the Chi-squared test. Logistic regression analyses were performed to assess the relationship between PCOS and asthma, adjusting for potential confounding covariates including age, BMI and smoking status. The selection of variables was based on identifying all measured clinical variables of known or suspected prognostic importance for the outcome of interest (and/or exhibiting $\mathrm{p}<0.1$ on univariate analysis). To further explore these relationships, the interaction between PCOS and BMI (as a continuous measurement) was examined on stratified analysis. All statistical analyses were survey weighted by area of residence for adjustment of intentional oversampling of this survey from rural and remote areas. All p-values were calculated from two-tailed tests of statistical significance with a type I error rate of $5 \%$. All analyses were performed using Stata version 12.0 (StataCorp, College Station, TX, USA).

\section{Results}

At Survey 4, prevalence of PCOS was 5.8\% (95\% CI 5.3-6.4\%) with 478 out of 8612 women reporting PCOS. The demographic characteristics of the study population are outlined in table 1 . Women with and without PCOS had similar baseline characteristics except higher BMI, as previously reported [17]. The use of OCP for any length of time was not significantly different between women with PCOS and women without PCOS (table 1).

Asthma was reported in $15.2 \%$ of women with PCOS compared with $10.6 \%$ of women without PCOS $(\mathrm{p}=0.004)$ (figure 2a). Women with PCOS who had asthma had a trend for a higher BMI compared with 
TABLE 1 Baseline characteristics in women with and without polycystic ovary syndrome (PCOS)

\begin{tabular}{|c|c|c|c|}
\hline & PCOS & Non-PCOS & p-value \\
\hline Subjects & 478 & 8134 & \\
\hline Age years & $30.5 \pm 0.1$ & $30.6 \pm 0.02$ & 0.12 \\
\hline Weight kg & $77.0 \pm 1.0$ & $69.1 \pm 0.2$ & $<0.001$ \\
\hline BMI $\mathrm{kg} \cdot \mathrm{m}^{-2}$ & $28.0 \pm 0.3$ & $25.1 \pm 0.1$ & $<0.001$ \\
\hline$<18.5$ & $14(3.6)$ & 291 (3.9) & 0.76 \\
\hline $18.5-24.9$ & $169(38.6)$ & 4347 (58.7) & $<0.001$ \\
\hline $25.0-29.9$ & $117(25.7)$ & $1780(22.4)$ & 0.13 \\
\hline$\geqslant 30.0$ & 149 (32.2) & $1245(15.1)$ & $<0.001$ \\
\hline Asthma & 77 (15.2) & $856(10.6)$ & 0.004 \\
\hline Smoking & 80 (16.5) & $398(19.0)$ & 0.22 \\
\hline \multicolumn{4}{|l|}{ OCP use years } \\
\hline No & 39 (8.0) & 686 (8.8) & 0.58 \\
\hline$<2$ & 51 (11.0) & $610(7.8)$ & 0.02 \\
\hline $2-6$ & $120(24.8)$ & $1934(24.1)$ & 0.75 \\
\hline$>6$ & $262(56.2)$ & $4857(59.2)$ & 0.22 \\
\hline \multicolumn{4}{|l|}{ Highest qualification } \\
\hline Year 12 or less & $116(22.6)$ & 2030 (21.5) & 0.60 \\
\hline Trade/certificate & $150(29.1)$ & $2228(26.2)$ & 0.19 \\
\hline University/higher university degree & $210(48.3)$ & $3848(52.3)$ & 0.12 \\
\hline \multicolumn{4}{|l|}{ Annual personal income AUD } \\
\hline$\leqslant 15999$ & $125(27.2)$ & $2117(26.1)$ & 0.65 \\
\hline $16000-51999$ & $196(42.0)$ & $3400(44.3)$ & 0.37 \\
\hline$\geqslant 52000$ & $126(30.8)$ & $1986(29.6)$ & 0.59 \\
\hline \multicolumn{4}{|l|}{ Occupation } \\
\hline No paid job & 90 (18.7) & $1565(18.3)$ & 0.84 \\
\hline Trades and services & 99 (19.9) & $1588(17.7)$ & 0.26 \\
\hline Advanced trades and services & $102(21.6)$ & $1577(19.9)$ & 0.41 \\
\hline Professional & $177(39.9)$ & $3243(44.1)$ & 0.10 \\
\hline
\end{tabular}

Data are presented as $n$, mean \pm SE or $n(\%)$, unless otherwise stated. BMI: body mass index; OCP: oral contraceptive pill. All estimates adjusted for area of residence to account for oversampling of women from rural and remote areas.

women without asthma (29.9 \pm 0.9 versus $\left.27.7 \pm 0.4 \mathrm{~kg} \cdot \mathrm{m}^{-2} ; \mathrm{p}=0.054\right)$ (figure $2 \mathrm{~b}$ ). Women without PCOS who had asthma had a higher BMI compared with women without asthma $\left(26.4 \pm 0.2\right.$ versus $24.9 \pm 0.1 \mathrm{~kg} \cdot \mathrm{m}^{-2}$; $\mathrm{p}<0.001$ ) (figure $2 \mathrm{~b}$ ).

Univariable and multivariable regression analyses

On univariable regression analysis, asthma was associated with PCOS (odds ratio (OR) 1.5, 95\% CI 1.14-2.0; $\mathrm{p}=0.004)$. Smoking and OCP use were not associated with asthma (table 2).
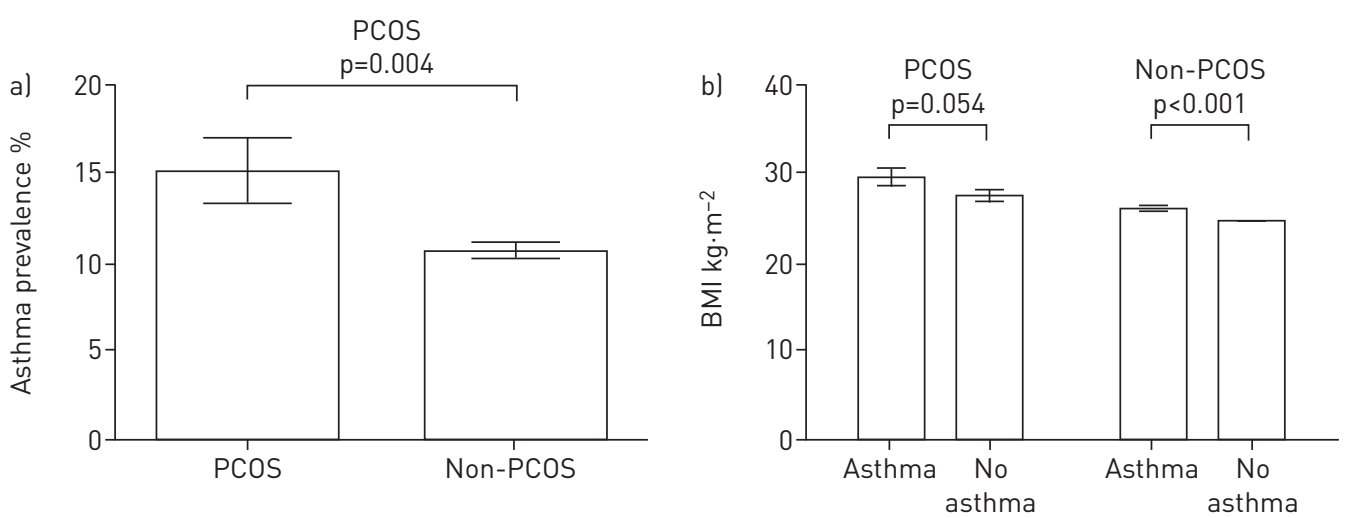

FIGURE 2 a) Asthma prevalence by polycystic ovary syndrome (PCOS) status. b) Body mass index (BMI) by asthma and PCOS status. Data are presented as mean \pm SE. 
TABLE 2 Univariable and multivariable regression of factors associated with asthma

\begin{tabular}{|c|c|c|c|c|}
\hline & \multicolumn{2}{|c|}{ Univariable regression } & \multicolumn{2}{|c|}{ Multivariable regression" } \\
\hline & OR $(95 \% \mathrm{CI})$ & p-value & OR $(95 \% \mathrm{CI})$ & p-value \\
\hline PCOS & $1.50(1.14-1.98)$ & 0.004 & $1.34(1.00-1.79)$ & 0.047 \\
\hline Age years & $0.94(0.89-0.99)$ & 0.02 & $0.94(0.89-0.99)$ & 0.01 \\
\hline \multicolumn{5}{|l|}{$\mathrm{BMI} \mathbf{k g} \cdot \mathrm{m}^{-2}$} \\
\hline$<18.5$ & $0.97(0.63-1.51)$ & 0.90 & $0.97(0.62-1.50)$ & 0.89 \\
\hline $18.5-24.9$ & \multicolumn{2}{|c|}{ Reference category } & \multicolumn{2}{|c|}{ Reference category } \\
\hline $25.0-29.9$ & $1.24(1.03-1.50)$ & 0.03 & $1.24(1.02-1.50)$ & 0.03 \\
\hline$\geqslant 30.0$ & $1.82(1.50-2.21)$ & $<0.001$ & $1.77(1.46-2.15)$ & $<0.001$ \\
\hline Smoking & $1.01(0.84-1.22)$ & 0.89 & $0.99(0.82-1.20)$ & 0.92 \\
\hline OCP use & $1.00(0.82-1.22)$ & 0.97 & & \\
\hline
\end{tabular}

PCOS: polycystic ovary syndrome; BMI: body mass index; OCP: oral contraceptive pill. ": adjusted for age, $\mathrm{BMI}$ and smoking status.

On multivariable analysis, the significant relationship between PCOS and asthma was maintained (adjusted OR 1.34, 95\% CI 1.00-1.79; p=0.047) (table 2). BMI in both the overweight and obese categories remained significantly associated with asthma after adjusting for PCOS status, age and smoking (table 2). There was no significant interaction between PCOS and BMI for the outcome of asthma $(p=0.09)$.

\section{Discussion}

To the best of our knowledge, there have been no community-based studies examining the association between asthma and PCOS in women of reproductive age. Here, we examined self-reported diagnosed asthma in women with PCOS compared with those without PCOS in a large community-based cohort of women aged 28-33 years, with specific focus on the relationship with PCOS status and BMI. Our findings also support previous evidence that increased BMI is associated with asthma. We advance knowledge in this field by reporting for the first time that PCOS status was associated with increased risk of asthma independently of BMI.

Our findings are in agreement with a retrospective study of women aged between 12 and 60 years from the Danish population, which reported an increased prevalence of asthma (3\% versus $2.2 \%$; $\mathrm{p}<0.001)$ and a higher usage of asthma medication $(19.2 \%$ versus $14.1 \%$; $<0.001)$ in age-matched women with and without PCOS [13]. It also supports a prior Australian study that showed a higher prevalence of asthma in pregnant women with PCOS compared with pregnant women without PCOS $(13.6 \%$ versus $9.9 \% ; \mathrm{p}<0.001)$ [6]. A retrospective cohort study of age-matched Australian women with and without PCOS ( $>15$ years old) similarly reported that women with PCOS were hospitalised more often for asthma compared with controls $(10.6 \%$ versus 4.5\%; $\mathrm{p}<0.001)$ [22]. In both the Danish and Australian studies, data on BMI was not available, and therefore we were not able to assess the relationship between PCOS, asthma and BMI. Here, we significantly extend the results of this previous research by reporting that while BMI was independently associated with asthma, the elevated prevalence of asthma in PCOS was maintained after adjustment for BMI. Our results therefore suggest an independent relationship between PCOS and asthma. There is no current literature to explain the exact mechanism underpinning this association.

A Northern European study by SvANEs et al. [23] reported that self-reported irregular menstruation was significantly associated with self-reported asthma, asthma symptoms and hay fever in women aged 25-42 years, but not in women aged $\geqslant 43$ years. It was hypothesised that sex hormone imbalances and metabolic risk factors underlying pathological irregular menstruation may be responsible for this association rather than the irregular menstruation per se [23]. A cross-sectional analysis of women aged 28-44 years from a European community reported that forced vital capacity (FVC) was significantly lower, and asthma, asthma symptoms and hay fever were more prevalent, in women with irregular menstruation at any level of BMI [19]. Previous comparative studies have shown abnormal oestradiol and/or progesterone levels in reproductive-aged women with asthma compared with women without asthma [24]. Furthermore, women with Turner's syndrome have significantly reduced bronchial reactivity after receiving oestrogen treatment [25]. Irregular menstruation and an altered sex hormone profile are major features of PCOS. The significant association of PCOS with asthma regardless of BMI found in our study further supports the previous literature that there may be a contributing role of sex hormones towards the pathogenesis of asthma in women [26-28].

The relationship between OCP use and asthma was explored in this study given that the existing evidence regarding the influence of OCP use on asthma symptoms is conflicting $[19,20]$. OCP use has been 
reported to be associated with increased asthma symptoms in women in normal and overweight BMI categories in a Nordic-Baltic population [19]. Conversely, SALAM et al. [20] reported that females with pre-existing asthma have less wheezing if they are using the OCP. However, these studies did not include the indication for OCP use, such as oligomenorrhoea, PCOS or contraceptive purposes. It is therefore not possible to clarify the potential contribution of PCOS to the observed association between OCP use and asthma in previous studies. As reported in our previous study on the ALSWH, OCP usage in women with PCOS was reduced compared with women without PCOS [29]. Our study did not show any significant association between OCP use and asthma on univariable analysis. Therefore, the association between OCP use and asthma, and the effect of this potential association on the relationship between PCOS and asthma, warrants further research using longitudinal studies to explore the possible influence of sex hormones on the pathophysiology of asthma.

We confirm previous reports of an elevated BMI in women with PCOS compared with those without PCOS $[8,30]$. Furthermore, we also report here that there is a trend for women with asthma to have a higher BMI in the PCOS group and a significantly higher BMI in the non-PCOS group. BMI in both overweight and obese categories was significantly associated with asthma, supporting previous evidence linking obesity to asthma $[11,15,16]$. A meta-analysis of prospective epidemiological studies demonstrated a dose-response relationship between increasing BMI and 1-year incident asthma (OR 1.51, 95\% CI $1.27-1.80 ; \mathrm{p}<0.001)$ [31]. The mechanism behind this association is unclear and several contributing factors have been postulated, such as genetics, hormonal influences, enhanced inflammatory response due to increased cytokine levels in obese patients leading to increased airway inflammation as well as the mechanical effect of obesity $[15,16,32,33]$. While we have shown here that PCOS is independently associated with asthma, this elevated BMI in PCOS may then further increase the prevalence of asthma compared with women without PCOS. We therefore hypothesise that further aetiological factors related to PCOS may contribute to the increased risk of asthma observed here and in prior research.

One of the well-documented metabolic complications of PCOS is insulin resistance and type 2 diabetes independent of BMI $[9,17]$. In a cross-sectional study of 3911 British females aged 60-79 years, forced expiratory volume in $1 \mathrm{~s}$ and lower FVC, which reflects lung volume, were inversely associated with insulin resistance and type 2 diabetes [34]. PCOS is also considered to be associated with chronic low-grade inflammation similar to obesity and as well as autoimmunity $[7,35,36]$. The underlying inflammatory process of PCOS may be contributing to airway inflammation and increased risk of asthma. As our study showed increased prevalence of asthma in women with PCOS, further longitudinal studies are warranted to explore whether the metabolic characteristics such as insulin resistance and/or inflammatory nature of PCOS are contributing to this association with asthma.

The strengths of our study include a large number of participants from a randomly selected community-based cohort representative of the general Australian population [21]. Although attrition occurred in this cohort study, it is reported to be of minimal impact on associations between variables [37]. The prevalence of self-reported PCOS in our study is lower than the prevalence of PCOS in the literature. This is most likely due to underdiagnosis of PCOS in the Australian population as reported by MARCH et al. [1] where an estimated $70 \%$ of Australian women with PCOS were not diagnosed, which may underestimate the association between PCOS and asthma. The limitation of our study is reliance on self-reported diagnosis of PCOS and asthma. No physical measures or biochemical samples were obtained. However, self-reported PCOS diagnosis in this survey had been previously validated against menstrual irregularities [38]. Self-reported asthma in previous studies had been shown to reflect physician diagnosed asthma accurately [39]. Self-reported BMI has also been validated to correlate with observed BMI by a recent study [40].

Our study has data regarding the proportion of women who have hay fever, allergies and sinusitis. However, the survey question for this data was nonspecific and cannot specify the atopic nature of the conditions. This lack of data on atopy is one of the limitations of our study. However, it is important to note that as atopy and asthma are closely related, the inclusion of atopy data may have a confounding effect on the outcome variable, i.e. asthma. Although our study has data on irregular periods, given the close relationship between irregular periods and PCOS, we have not included irregular periods as an explanatory variable $[2,3,5]$. Our study did not have data on the severity of asthma and the type of asthma treatment, and the data for physical activity and mood disorders were not included. This is also an observational study only, and cannot be used to establish causation between PCOS, BMI and asthma.

In conclusion, we report here for the first time the potential link between asthma, PCOS and BMI. We report an increased prevalence of asthma in women with PCOS, and an independent association of both PCOS and BMI with increased risk of asthma. The pathophysiology underlying PCOS and asthma is likely multifactorial, and further prospective longitudinal population-based studies are required to clarity the complex interplay between hormonal, metabolic and inflammatory factors underlying asthma, PCOS 
and BMI. The insight provided by our study advances our current understanding of the wider health implications of asthma and PCOS. This knowledge will provide us with a broader range of screening strategies for women with asthma and PCOS, and their associated comorbidities. It also directs our future approach to implementing a more comprehensive management for asthmatic women with PCOS.

\section{Acknowledgements}

The research on which this paper is based was conducted as part of the Australian Longitudinal Study on Women's Health undertaken by the University of Newcastle (Callaghan, Australia) and University of Queensland (St Lucia, Australia). We are grateful to the Australian Government Dept of Health for funding and to the women who provided the survey data.

Author contributions: T.D. Htet: literature review and synthesis, critical discussion, drafting and revision of manuscript; H.J. Teede: critical discussion, revision of manuscript; B. de Courten: drafting and revision of manuscript; D. Loxton: data acquisition, drafting and revision of manuscript; F.G. Real: drafting and revision of manuscript; L.J. Moran: critical discussion, drafting and revision of manuscript; A.E. Joham: detailed data analysis, critical discussion, drafting and revision of manuscript.

\section{References}

1 March WA, Moore VM, Willson KJ, et al. The prevalence of polycystic ovary syndrome in a community sample assessed under contrasting diagnostic criteria. Hum Reprod 2010; 25: 544-551.

2 Sirmans SM, Pate KA. Epidemiology, diagnosis, and management of polycystic ovary syndrome. Clin Epidemiol 2013; 6: 1-13.

3 Rotterdam ESHRE/ASRM-Sponsored PCOS Consensus Workshop Group. Revised 2003 consensus on diagnostic criteria and long-term health risks related to polycystic ovary syndrome (PCOS). Hum Reprod 2004; 19: 41-47.

4 Azziz R, Carmina E, Dewailly D, et al. The Androgen Excess and PCOS Society criteria for the polycystic ovary syndrome: the complete task force report. Fertil Steril 2009; 91: 456-488.

5 Coffey S, Bano G, Mason HD. Health related quality of life in women with polycystic ovary syndrome: a comparison with general population using the Polycystic Ovary Syndrome Questionnaire (PCOSQ) and Short From-36 (SF-36). Gynaecol Endocrinol 2006; 22: 80-86.

6 Doherty DA, Newnham JP, Bower C, et al. Implications of polycystic ovary syndrome for pregnancy and for the health of offspring. Obstet Gynecol 2015; 125: 1397-1406.

7 Randeva HS, Tan BK, Weickert MO, et al. Cardiometabolic aspects of the polycystic ovary syndrome. Endocr Rev 2012; 33: 812-841.

8 Ollila MM, Piltonen T, Puukka K, et al. Weight gain and dyslipidemia in early adulthood associate with polycystic ovary syndrome: prospective cohort study. J Clin Endocrinol Metab 2016; 101: 739-747.

9 Teede HJ, Hutchison S, Zoungas S, et al. Insulin resistance, the metabolic syndrome, diabetes, and cardiovascular disease risk in women with PCOS. Endocrine 2006; 30: 45-53.

10 Beasley R, Semprini A, Mitchell EA. Risk factors for asthma: is prevention possible? Lancet 2015; 386: 1075-1085.

11 Toskala E, Kennedy DW. Asthma risk factors. Int Forum Allergy Rhinol 2015; 5: Suppl. 1, S11-S16.

12 Akinbami LJ, Simon AE, Rossen LM. Changing trends in asthma prevalence among children. Pediatrics 2016; 137: $1-7$.

13 Glintborg D, Hass Rubin K, Nybo M, et al. Morbidity and medicine prescriptions in a nationwide Danish population of patients diagnosed with polycystic ovary syndrome. Eur J Endocrinol 2015; 172: 627-638.

14 Lim SS, Davies MJ, Norman RJ, et al. Overweight, obesity and central obesity in women with polycystic ovary syndrome: a systematic review and meta-analysis. Hum Reprod Update 2012; 18: 618-637.

15 Sutherland ER. Linking obesity and asthma. Ann NY Acad Sci 2014; 1311: 31-41.

16 Weiss ST. Obesity: insight into the origins of asthma. Nat Immunol 2005; 6: 537-539.

17 Joham AE, Ranasinha S, Zoungas S, et al. Gestational diabetes and type 2 diabetes in reproductive-aged women with polycystic ovary syndrome. J Clin Endocrinol Metab 2014; 99: E447-E452.

18 Real FG, Svanes C, Omenaas ER, et al. Menstrual irregularity and asthma and lung function. J Allergy Clin Immunol 2007; 120: 557-564.

19 Macsali F, Real FG, Omenaas ER, et al. Oral contraception, body mass index, and asthma: a cross-sectional Nordic-Baltic population survey. J Allergy Clin Immunol 2009; 123: 391-397.

20 Salam MT, Wenten M, Gilliland FD. Endogenous and exogenous sex steroid hormones and asthma and wheeze in young women. J Allergy Clin Immunol 2006; 117: 1001-1007.

21 Lee C, Dobson AJ, Brown WJ, et al. Cohort profile: the Australian Longitudinal Study on Women's Health. Int J Epidemiol 2005; 34: 987-991.

22 Hart R, Doherty DA. The potential implications of a PCOS diagnosis on a woman's long-term health using data linkage. J Clin Endocrinol Metab 2015; 100: 911-919.

23 Svanes C, Real FG, Gislason T, et al. Association of asthma and hay fever with irregular menstruation. Thorax 2005; 60: 445-450.

24 Rubio RL, Rodriguez BG, Collazzo JJA, et al. Comparative study of progesterone, estradiol and cortisol concentrations in asthmatic and non-asthmatic women. Allergol Immunopathol 1988; 16: 263-266.

25 Villa MP, Bernardi F, Burnaccini M, et al. Bronchial reactivity and sex hormone: study in a Turner's population. Pediatr Pulmonol 1990; 9: 199-205.

26 Becklake MR, Kauffmann F. Gender differences in airway behaviour over the human life span. Thorax 1999; 54: $1119-1138$

27 Macsali F, Svanes C, Bjørge L, et al. Respiratory health in women: from menarche to menopause. Expert Rev Respir Med 2012; 6: 187-202.

28 Leynaert B, Sunyer J, Garcia-Esteban R, et al. Gender differences in prevalence, diagnosis and incidence of allergic and non-allergic asthma: a population-based cohort. Thorax 2012; 67: 625-631. 
29 Joham AE, Boyle JA, Ranasinha S, et al. Contraception use and pregnancy outcomes in women with polycystic ovary syndrome: data from the Australian Longitudinal Study on Women's Health. Hum Reprod 2014; 29: 802-808.

30 Morgan CL, Jenkins-Jones S, Currie CJ, et al. Evaluation of adverse outcome in young women with polycystic ovary syndrome versus matched, reference controls: a retrospective, observational study. J Clin Endocrinol Metab 2012; 97: 3251-3260.

31 Beuther DA, Sutherland ER. Overweight, obesity, and incident asthma: a meta-analysis of prospective epidemiologic studies. Am J Respir Crit Care Med 2007; 175: 661-666.

32 Chen YP, Zhang JH, Li CQ, et al. Obesity enhances Th2 inflammatory response via natural killer T cells in a murine model of allergic asthma. Int J Clin Exp Med 2015; 8: 15403-15412.

33 Coffey MJ, Torretti B, Mancuso P. Adipokines and cysteinyl leukotrienes in the pathogenesis of asthma. J Allergy 2015; 2015: 157919.

34 Lawlor DA, Ebrahim S, Smith GD. Associations of measures of lung function with insulin resistance and Type 2 diabetes: findings from the British Women's Heart and Health Study. Diabetologia 2004; 47: 195-203.

35 Hefler-Frischmuth K, Walch K, Huebl W, et al. Serologic markers of autoimmunity in women with polycystic ovary syndrome. Fertil Steril 2010; 93: 2291-2294.

36 Kelly CCJ, Lyall H, Petrie JR, et al. Low grade chronic inflammation in women with polycystic ovarian syndrome. J Clin Endocrinol Metab 2001; 86: 2453-2455.

37 Powers J, Loxton D. The impact of attrition in an 11-year prospective longitudinal study of younger women. Ann Epidemiol 2010; 20: 318-321.

38 Teede HJ, Joham AE, Paul E, et al. Longitudinal weight gain in women identified with polycystic ovary syndrome: results of an observational study in young women. Obesity 2013; 21: 1526-1532.

39 Toren K, Brisman J, Jarvholm B. Asthma and asthma-like symptoms in adults assessed by questionnaires. A literature review. Chest 1993; 104: 600-608.

40 Burton NW, Brown W, Dobson A. Accuracy of body mass index estimated from self-reported height and weight in mid-aged Australian women. Aust NZ J Public Health 2010; 34: 620-623. 